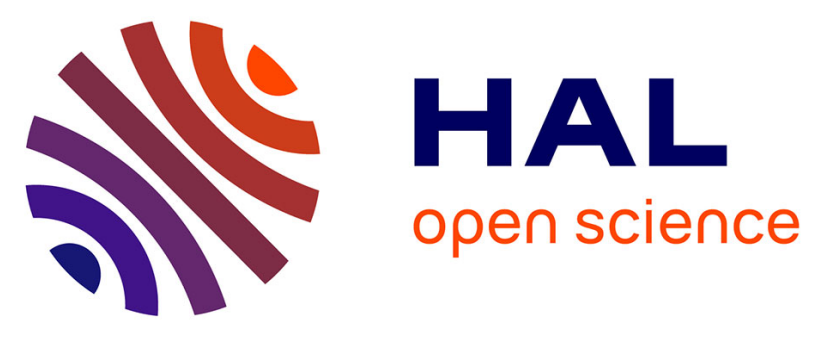

\title{
Next-generation sequencing confirms the implication of SLC24A1 in autosomal-recessive congenital stationary night blindness
}

Marion Neuillé, Sivasankar Malaichamy, Maria Vadalà, Christelle Michiels, Christel Condroyer, Ramya Sachidanandam, Sundaramurthy Srilekha, Tharigopala Arokiasamy, Mélanie Letexier, Vanessa Démontant, et al.

\section{To cite this version:}

Marion Neuillé, Sivasankar Malaichamy, Maria Vadalà, Christelle Michiels, Christel Condroyer, et al.. Next-generation sequencing confirms the implication of SLC24A1 in autosomal-recessive congenital stationary night blindness. Clinical Epigenetics, 2016, 10.1111/cge.12746 . hal-01285951

\section{HAL Id: hal-01285951 \\ https://hal.sorbonne-universite.fr/hal-01285951}

Submitted on 10 Mar 2016

HAL is a multi-disciplinary open access archive for the deposit and dissemination of scientific research documents, whether they are published or not. The documents may come from teaching and research institutions in France or abroad, or from public or private research centers.
L'archive ouverte pluridisciplinaire HAL, est destinée au dépôt et à la diffusion de documents scientifiques de niveau recherche, publiés ou non, émanant des établissements d'enseignement et de recherche français ou étrangers, des laboratoires publics ou privés. 


\section{NOVEL GENE DISCOVERIES}

\section{Next-generation sequencing confirms the implication of $S L C 24 A 1$ in autosomal-recessive}

congenital stationary night blindness (CSNB)

Marion Neuillé ${ }^{1}$, Sivasankar Malaichamy ${ }^{2}$, Maria Vadalà ${ }^{3}$, Christelle Michiels ${ }^{1}$, Christel Condroyer $^{1}$, Ramya Sachidanandam ${ }^{4}$, Sundaramurthy Srilekha ${ }^{2}$, Tharigopala Arokiasamy ${ }^{2}$, Mélanie Letexier ${ }^{5}$, Vanessa Démontant ${ }^{1}$, José-Alain Sahel ${ }^{1,6,7,8,9}$, Parveen Sen $^{10}$, Isabelle Audo ${ }^{1,6,7}$, Nagasamy Soumittra ${ }^{2}$, Christina Zeitz ${ }^{1 *}$

${ }^{1}$ Sorbonne Universités, UPMC Univ Paris 06, INSERM, CNRS, Institut de la Vision, 17 rue Moreau, 75012 Paris, France;

${ }^{2}$ SNONGC Department of Genetics and Molecular Biology, Vision Research Foundation, Chennai, India;

${ }^{3}$ Department of Experimental Medicine and Clinical Neuroscience, Ophthalmology Section, University of Palermo, Italy;

${ }^{4}$ Department of Optometry, Medical Research Foundation, Chennai, India;

${ }^{5}$ IntregraGen SA, Evry, F-91000, France;

${ }^{6}$ CHNO des Quinze-Vingts, DHU Sight Restore, INSERM-DHOS CIC 1423, 28 rue de Charenton, 75012 Paris, France;

${ }^{7}$ University College of London, Institute of Ophthalmology, London, EC1V 9EL, UK;

${ }^{8}$ Fondation Ophtalmologique Adolphe de Rothschild, Paris, F-75012, France;

${ }^{9}$ Académie des Sciences-Institut de France, Paris, F-75006, France;

${ }^{10}$ Department of Vitreo-Retinal Services, Medical Research Foundation, Chennai, India.

*Corresponding author: Christina Zeitz

Institut de la Vision

Department of Genetics 
17, Rue Moreau

75012 Paris

France

Email:christina.zeitz@inserm.fr

Phone: +33153462540

FAX: +33153462602

\section{Conflict of interest statement}

The funders had no role in study design, data collection, analysis and interpretation, decision to publish, or preparation of the manuscript. The authors declare no competing financial interests.

\section{Aknowledgments}

The authors are grateful to patients and families to participate in the study. The project was supported by Fondation Voir et Entendre (CZ), Prix Dalloz for "la recherche en ophtalmologie" (CZ), Fondation pour la Recherche Médicale (FRM DVS20131228918) in partnership with Fondation Roland Bailly (CZ), Fédération des Aveugles et Amblyopes de France $(\mathrm{MN})$, Ville de Paris and Région Ile de France, LABEX LIFESENSES [reference ANR-10-LABX-65] supported by French state funds managed by the Agence Nationale de la Recherche within the Investissements d'Avenir programme [ANR-11-IDEX-0004-0], Foundation Fighting Blindness center grant [C-CMM-0907-0428-INSERM04] and by the Indian Council of Medical Research and INSERM (France), an Indo-French collaborative program (No: 53/1/Indo-Foreign/Oph/10-NCD-II). 


\section{GRAPHICAL ABSTRACT}

\section{Position of disease causing variants in SLC24A1 underlying CSNB}

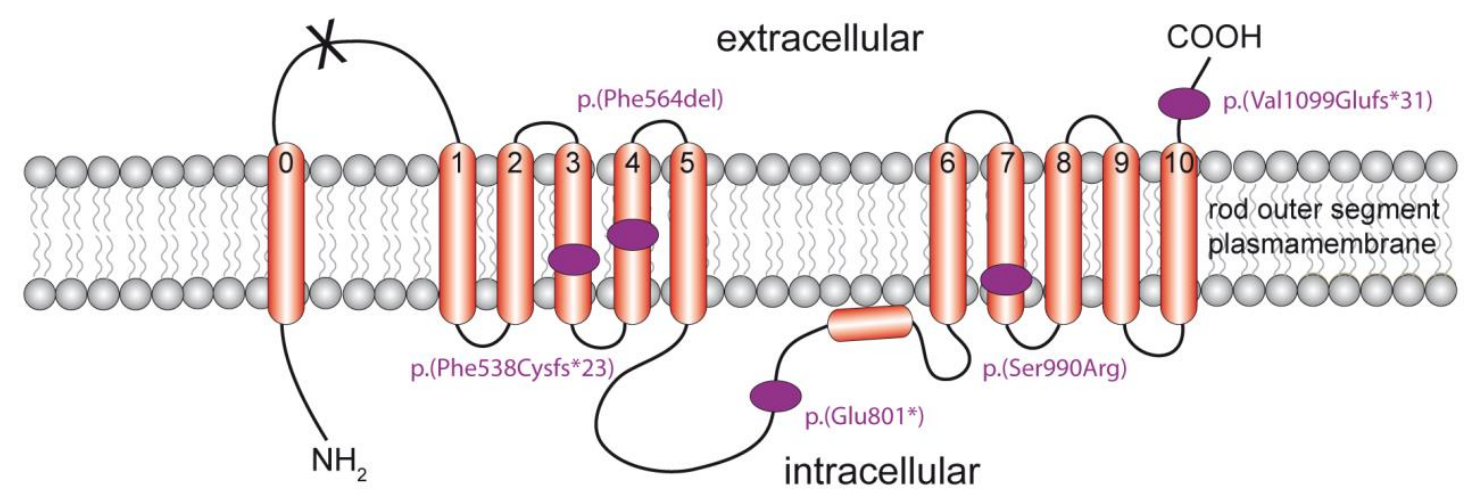

\section{ABSTRACT}

Congenital stationary night blindness (CSNB) is a clinically and genetically heterogeneous retinal disorder which represents rod photoreceptor dysfunction or signal transmission defect from photoreceptors to adjacent bipolar cells. Patients displaying photoreceptor dysfunction show a Riggs-electroretinogram (ERG) while patients with a signal transmission defect show a Schubert-Bornschein-ERG. The latter group is subdivided into complete or incomplete (ic) CSNB. Only few CSNB cases with Riggs-ERG and only one family with a disease causing variant in SLC24Al have been reported. Whole-exome sequencing (WES) in a previously diagnosed icCSNB patient identified a homozygous nonsense variant in SLC24A1. Indeed, reinvestigation of the clinical data corrected the diagnosis to Riggs-form of CSNB. Targeted next-generation sequencing (NGS) identified compound heterozygous deletions and a homozygous missense variant in SLC24Al in two other patients, respectively. ERG abnormalities varied in these three cases but all patients had normal visual acuity, no myopia or nystagmus, unlike in Schubert-Bornschein-type of CSNB. This confirms that SLC24A1 defects lead to CSNB, and outlines phenotype/genotype correlations in CSNB subtypes. In case of unclear clinical characteristics, NGS techniques are helpful to clarify the diagnosis. 
Key Words

Congenital stationary night blindness; high-throughput sequencing; humans; SLC24A1 


\section{INTRODUCTION}

Congenital stationary night blindness (CSNB) is a clinically and genetically heterogeneous group of retinal disorders caused by variants in genes implicated in the phototransduction cascade or in retinal signaling from photoreceptors to adjacent bipolar cells $(1,2)$.

Most individuals affected with CSNB show characteristic electroretinogram (ERG) abnormalities, named after Schubert and Bornschein who initially reported them, in which the b-wave amplitude is smaller than that of the a-wave, which is globally normal, in the darkadapted bright flash condition (3). This electronegative waveform can be divided in two subtypes, complete (c)CSNB [MIM 310500, MIM 257270, MIM 613216, MIM 614565, MIM 615058] and incomplete (ic)CSNB [MIM 300071] (4). Patients suffering from the SchubertBornschein-type of CSNB often show ocular abnormalities in addition to night blindness, including reduced visual acuity, photophobia, myopia, nystagmus and strabismus $(1,2)$. cCSNB is characterized by a drastically reduced rod b-wave response due to ON-bipolar cell dysfunction, and specific cone ERG waveforms (5). cCSNB has been associated with disease causing variants in NYX [MIM 300278] (6, 7), GRM6 [MIM 604096] (8, 9), TRPM1 [MIM 603576] (10-12), GPR179 [MIM 614515] (13, 14) and LRIT3 [MIM 615004] (15), whose products localize at the dendritic tips of ON-bipolar cells (2). icCSNB is characterized by a reduced rod b-wave and substantially reduced cone responses, indicative of both $\mathrm{ON}$ - and OFF-bipolar cell dysfunction. icCSNB has been associated with disease causing variants in CACNA1F [MIM 300110] (16, 17), CABP4 [MIM 608965] (18) and CACNA2D4 [MIM 608171] (19), genes coding for proteins localized presynaptically and important for the continuous glutamate release at the photoreceptor synapse $(1,2)$.

Less commonly, individuals affected with CSNB display another type of ERG abnormalities, reported by Riggs (20) [MIM 610445, MIM 610444, MIM 616389, MIM 163500, MIM 
613830]: in these cases there is a generalized rod photoreceptor dysfunction, dark adapted responses being dominated by cone responses which are normal. Therefore, the a-wave amplitude is reduced in response to a bright flash under dark adaptation with a possible additional reduction of the b/a ratio and an electronegative waveform due to the photopic hill phenomenon characteristic of the cone pathway function (21). Photopic ERG responses are preserved due to the normal cone system function. These CSNB patients manifest distinct clinical characteristics from the Schubert-Bornschein-patients, including a normal visual acuity and no clinical signs of myopia and/or nystagmus $(1,2)$. Riggs-type of CSNB has been associated with disease causing variants in RHO [MIM 180380] (22), GNAT1 [MIM 139330] (23), PDE6B [MIM 180072] (24, 25) and SLC24A1 [MIM 603617] (26), underlying autosomal dominant (RHO, GNAT1 and PDE6B) or autosomal recessive (GNAT1, SLC24A1) CSNB. Only four families with four different disease causing variants in $R H O(22,27-29)$, three families with three different disease causing variants in $\operatorname{GNAT1}(23,30,31)$, two families with two different disease causing variants in $\operatorname{PDE6B}(25,32)$ and one family with one disease causing variant in SLC24Al (26) were described. The corresponding proteins localize in rods and have a role in their phototransduction cascade (2).

To date, more than 360 different disease causing variants have been identified in genes underlying CSNB (2). However, despite comprehensive genotyping studies on our own CSNB cohort, some cases carried no disease causing variants in known genes underlying CSNB. This is a strong indication that disease causing variants in other genes remain to be discovered or that defects in regulatory elements or introns might be involved. In addition, the phenotype may not be well characterized or one phenotype may be associated with different gene defects.

Disease causing variants in many genes associated with CSNB have been identified through classical linkage approaches $(6,7,16,17,25,26)$ or through a candidate gene approach 
comparing the human phenotype to similar phenotypes observed in knockout or naturally occurring animal models $(8-12,14,18,22,33)$. One limitation of the linkage approach is the requirement to examine large families. Techniques using massively parallel sequencing of known human exons implicated in inherited retinal disorders and all human exons, also called whole exome sequencing (WES) have recently been successful in identifying disease causing variants in known and novel genes underlying heterogeneous diseases $(34,35)$, including rodcone dystrophy $(34,36,37)$, cone or cone-rod dystrophy (38) and CSNB (13, 15, 34). Especially, WES is an unbiased method to identify the gene defect in families with unclear phenotypes and with only few family members available.

The aim of this study was to identify the gene defect in a previously diagnosed icCSNB family lacking disease causing variants in the known genes $C A C N A 1 F, C A B P 4, C A C N A 2 D 4$ and in a candidate gene $C A C N 2 B$ [MIM 600003], and in two other families with unclassified CSNB, never investigated before. 


\section{MATERIALS AND METHODS}

Methods for whole exome sequencing (WES), targeted next-generation sequencing (NGS) and investigation of annotated sequencing data are provided as supplementary data.

\section{Ethics statements}

Research procedures were conducted in accordance with institutional guidelines and the Declaration of Helsinki. Prior to genetic testing, informed consent was obtained from all CSNB-affected individuals and their family members.

\section{Clinical investigation}

Ophthalmic examination included best corrected Snellen visual acuity measurement, fundoscopy, D15 Farnworth color vision test, full-field ERG incorporating the International Society for Clinical Electrophysiology of Vision (ISCEV) standards (39), fundus autofluorescence (FAF), and spectral-domain optical coherence tomography (SD-OCT) (the extent of investigation depended on the referring center).

\section{$\underline{\text { Previous molecular genetic analysis }}$}

The DNA of the index patient of Family 1 was directly Sanger sequenced for the coding exonic and flanking intronic regions of the known genes underlying icCSNB and a candidate gene (CACNA1F, NM_005183.3; CABP4, NM_145200.3; CACNA2D4, NM_172364.4; CACNB2, NM_000724.3).

\section{$\underline{\text { Disease causing variants }}$}

Novel disease causing variants identified in SLC24A1 in this study have been deposited in the “Leiden Open Variation Database” (http://www.lovd.nl/3.0/home) prior to publication. 


\section{RESULTS}

The two male patients of Family 1 were previously reported to us as affected with icCSNB (Fig.1A). However, mutation analysis by Sanger sequencing in these cases in known genes underlying icCSNB and a candidate gene (CACNA1F, CACNA2D4,CABP4 and CACN2B) did not reveal any disease causing variant (data not shown). Subsequently, WES was performed. The index cases of Family 2 and 3 were screened for variants in all genes previously associated with CSNB by targeted NGS.

Applying our stringent criteria and filters to the sequencing data of the WES for Family 1, we reduced the number of putative variants from 1059 insertions/deletions (InDels) and 18,058 single nucleotide variants (SNVs) to four hemizygous variants in four genes, six compound heterozygous variants in three genes and one homozygous variant in one gene (Table S1 and Table S2). Due to the filtering and the pathogenic prediction, the most likely disease causing variant was the homozygous nonsense variant, c.2401G>T (p.(Glu801*)) in SLC24A1 (Fig.1A, Fig.2B and Table S2). The overall sequencing coverage of the captured regions in the family was $95 \%$ and $87.75 \%$ for a $10 \times$ and $25 \times$ depth of coverage, respectively, resulting in a mean sequencing depth of $77.75 \times$ per base (Table S3). Both unaffected parents were found to be heterozygous for this variant. The nucleotide T was read 104 and 74 times in the mother and father, respectively, whereas the G was found 97 and 101 times, respectively. The two affected boys showed 170 and 212 times the nucleotide T. Sanger sequencing confirmed these results (Fig.1B). The detailed clinical data of the index case of Family 1 were only available after $S L C 24 A 1$ defect identification. For this six year-old patient, night blindness was reported since early childhood. Visual acuity was 20/20 for both eyes, with no signs of myopia or nystagmus. Fundus examination revealed no retinal or disc abnormalities. Color sensitivity was normal. Scotopic ERG responses were severely reduced for both a- and bwaves for both eyes, whereas photopic responses were normal. 
Applying our stringent filtering criteria to the sequencing data of the targeted NGS from two unclassified CSNB families never genetically investigated before and considering the pathogenic variant predictions, we found that the index patient of Family 2 carried compound heterozygous deletions, c.1691_1693delTCT (p.(Phe564del)) and c.3291_3294delATCT (p.(Val1099Glufs*31))(Fig.1A, Fig.2B and Table S2) and that the index patient of the consanguineous Family 3 harbored a homozygous missense variant, c.2968A>C (p.(Ser990Arg)) affecting the well conserved amino acid residue Ser at position 990 in SLC24A1 (Fig.1A, Fig.2 and Table S2). For Family 2, the overall sequencing coverage of the captured regions was $100 \%$ for both $10 \times$ and $25 \times$ depth of coverage, resulting in a mean sequencing depth of $200 \times$ per base. For Family 3, the overall sequencing coverage of the captured regions was $100 \%$ for both $10 \times$ and $25 \times$ depth of coverage, resulting in a mean sequencing depth of $204 \times$ per base (Table S4). All these variants co-segregated with the phenotype (Fig.1) and were absent in 100 healthy ethnically matched control individuals. For the index patients of Family 2 and 3, night blindness was reported since early childhood. At the time of clinical examination, they were thirty two and thirty six years old, respectively. There were no signs of reduced visual acuity, myopia or nystagmus. Color vision was normal. Fundus examination revealed no retinal or disc abnormalities (Figs.3A and 3B). No retinal degeneration was detected by SD-OCT (Figs.3C and 3D). Scotopic ERG amplitudes were severely reduced in both patients with a more severe defect in the index patient of Family 3 (Fig.4 and Table 1). The index patient of Family 2 displays minimal decreased amplitudes in response to a single photopic flash with moderately reduced flicker responses (Fig.4 and Table 1). Photopic responses of the index patient of Family 3 are more altered with the amplitude of the b-wave which is significantly reduced in response to both the single flash as well as flicker (Fig.4 and Table 1).

\section{DISCUSSION}


In this work, we have used WES to identify a novel nonsense variant in SLC24A1 in a family previously reported to us as affected with icCSNB (Family 1). In addition, targeted NGS has led to the identification of three other novel variants in the same gene in two other unclassified CSNB families (Family 2 and 3).

The index patient and his affected brother of Family 1 carry a homozygous SNV in SLC24A1 (c.2401G>T). Localized in exon 7 , this variant is predicted to result in a truncated protein (p.(Glu801*)) or in nonsense mediated mRNA decay (NMD). The variant co-segregates with the phenotype as the affected brother is also homozygous and both non-affected parents are heterozygous for this variant. Interestingly, a SLC24Al disease causing variant had already been described in CSNB (c.1613_1614del, p.(Phe538Cysfs*23)). However, this disease causing variant was not associated with the incomplete form but with the Riggs-type of CSNB (26). On the clinical data on which we did not have access before genetic screening, myopia or nystagmus were not present and visual acuity was normal. In addition, it was noted that scotopic ERG responses were severely reduced, while photopic responses were in the normal range. These basic phenotypic descriptions are actually more in accordance with the Riggstype of CSNB than with icCSNB. However, the misdiagnosis from the referring clinician may have come from some icCSNB cases with disease causing variants in CACNA1F described with reduced scotopic a-wave $(40,41)$, but in these cases, photopic responses were also altered. Unfortunately the patient was no longer available for retesting to confirm a Riggstype of CSNB. Indeed, if we had access to these clinical details before genetic testing, SLC24A1 would have been a good candidate to screen first before the unbiased WES approach. In Family 2, we analyzed targeted NGS data of one index male and performed cosegregation analysis in his unaffected mother and one of his unaffected sisters, which led to the identification of compound heterozygous disease-causing deletions in SLC24Al (c.1691_1693delTCT and c.3291_3294delATCT). Localized in exons 2 and 10, these disease 
causing variants are predicted to result in a deletion of a phenylalanine (p.(Phe564del)) and a longer protein at the C-terminus (p.(Val1099Glufs*31)), respectively. In Family 3, we analyzed targeted NGS data of one index male in a family with consanguinity. We found a homozygous missense variant in SLC24Al (c.2968A>C), localized in exon 9, which cosegregates with the phenotype, resulting in the replacement of a serine by an arginine (p.(Ser990Arg)). For the index patients of Family 2 and 3, ERGs were not conclusive as scotopic as well as photopic responses were altered. In general, Riggs-ERG is characterized by a drastically reduced scotopic a-wave and b-wave due to rod dysfunction and normal photopic responses (20). This ERG phenotype is found in most of the Riggs-type of CSNB described, with disease causing variants in RHO, GNAT1 and PDE6B (22, 23, 25-28, 32). However, in some cases with disease causing variants in GNAT1, photopic responses were normal to moderately decreased in amplitudes $(30,31)$. Moreover, in the same family with the same SLC24Al disease causing variant, two indexes showed normal cone responses while they were modestly reduced in two other affected patients (26). It is unclear why photopic responses may be abnormal in Riggs-ERG since genes underlying the Riggs-type of CSNB are specifically expressed in rods. Some explanations may come from the fact that depending of the publications, not all ERGs were recorded according to the ISCEV current recommendations. Another explanation may come from interaction between rod and cone circuitry at the level of the inner retina or in rare cases the possibility for mild rod photoreceptor degeneration that may induce additional cone degeneration. However, in a Slc24al knock-out mouse model, recently published, despite slow rod degeneration, the number of cones was maintained as well as cone responses to light stimulations, indicating that cone-mediated vision is not compromised in this mouse model (42). Further investigations are needed to address this point. However, it is important to notice that myopia and nystagmus were also absent in index patients of Family 2 and 3 and that their visual 
acuity was normal. These data demonstrate that the ERG phenotype may diverge from the general characterization for the Riggs-type of CSNB but that absence of other ocular abnormalities such as reduced visual acuity, myopia or nystagmus are consistent findings upon clinical examination. On the contrary, if the phenotype may vary among icCSNB patients even within the same family, all affected patients have reduced visual acuity and variable degrees of abnormal refractive errors and nystagmus (43). In conclusion, if a CSNB phenotype is suspected in a patient on the basis of night blindness and no sign of retinal degeneration on retinal examination and if the ERG recording does not allow the distinction between Riggs and Schubert-Bornschein CSNB, variants in genes underlying Riggs-type of CSNB may cause the disease in case of normal visual acuity and absence of major refractive errors or nystagmus. Of note, fifty patients with unclear phenotype screened in our laboratory for disease causing variants in SLC24A1 did not reveal any disease causing variant (data not shown), indicating that this phenotype or gene defect is rare or underdiagnosed.

As mentioned above, in 2010, Riazuddin and coworkers reported the first case of RiggsCSNB associated with an autosomal-recessive inheritance. They found a homozygous $2 \mathrm{bp}$ deletion in exon 2 of SLC24A1 (c.1613_1614del), which is predicted to result in a frameshift leading to either truncated protein (p.(Phe538Cysfs*23)) or NMD (26). SLC24A1 is a $\mathrm{Na} / \mathrm{Ca}^{2}-\mathrm{K}$ exchanger which is present in the retina at the plasma membrane of the rod outer segments $(42,44,45)$. Under dark conditions, guanosine 3',5'-cyclic phosphate (cGMP)gated channels are opened and mediate an influx of cations, including $\mathrm{Ca}^{2+}$, (the so-called "dark current") into the cell (46). SLC24A1 balances this $\mathrm{Ca}^{2+}$ current by exchange of one $\mathrm{Ca}^{2+}$ against four $\mathrm{Na}^{+}$and one $\mathrm{K}^{+}(42)$. Illumination and activation of the phototransduction cascade closes cGMP-gated channels, leading to a lowering of intracellular free $\mathrm{Ca}^{2+}$. Finally, this low cellular $\mathrm{Ca}^{2+}$ concentration activates a negative feedback loop which results in the 
termination of the phototransduction and subsequently in the re-opening of cGMP-gated channels (45).

Recently, Vinberg et al. described a mouse model lacking Slc24al which mimics the pathophysiology of the Riggs-CSNB described in human (42). They confirmed localization of SLC24A1 to the plasma membrane of rod outer segments. Slc $24 a 1^{-/}$mice revealed a slow progressive retinal degeneration and displayed a less sensitive and slower rod-mediated vision with drastically reduced ERG a-wave amplitude under dark-adaptation. Therefore, despite dramatically decreased rod response amplitudes, rods are viable and still contribute to visual function and rod-mediated synaptic signaling. Moreover, light adaptation of rods is not significantly compromised indicating that there is an unknown mechanism for $\mathrm{Ca}^{2+}$ to be extruded from rod outer segments in the absence of SLC24A1, even if this mechanism is too slow to provide normal feedback to the phototransduction cascade in the time scale of dim flash responses. The capability of rods to regulate $\mathrm{Ca}^{2+}$ influx in the absence of SLC24A1 together with the reduced "dark-current" observed explain why there is no severe rod degeneration in this mouse model and are consistent with the stationary nature of the disorder. In our study, none of our patients demonstrated retinal degeneration at the time of the first investigation but this retinal degeneration has been reported to be slow and progressive in the Slc24al knock-out mouse model (42). Therefore, re-investigation of the patients with SLC24A1 disease causing variants may be useful to see if the retinal degeneration can also be observed. Moreover, a novel homozygous truncating disease causing variant has been reported in GNAT1 (c.904C>T, p. $\left.\left(\mathrm{Gln} 302^{*}\right)\right)$ in an 80 year-old patient who displays marked peripheral pigmentary deposits resembling bone spicule on fundus examination (47). This last observation is more in accordance with a slow and late onset form of retinitis pigmentosa than CSNB. Moreover, Gnatl lacking mouse model showed a subtle and slow thinning of the retina (48). 
Structurally, SLC24A1 is predicted to contain two clusters of five transmembrane domains (TM) that are separated by a large hydrophilic loop located in the cytosol. One putative TM does not span the membrane and precedes the second cluster of TM. An additional TM is present at the N-terminal of the protein but it is a signal peptide that can be cleaved. Therefore, $\mathrm{N}$ - and C-terminus are extracellular. The two TM clusters are proposed to constitute the two ion exchanger domains $(45,49)$. The novel disease causing variant identified by WES is located in the intracellular loop located between these two predicted ion exchanger domains (Fig.2B). Moreover, the deletion of the phenylalanine in position 564 as well as the replacement of a serine by an arginine in position 990 identified by targeted NGS are located in TM 4 and 7, respectively, and could alter the ion exchange function (Fig.2B). In particular, the Ser990 is analogous to Ser552 present in NCKX2 that appears to be critical for cation binding and transport $(50,51)$. It is hypothesized that the complete or even partial loss of the ion exchange function would result in abnormal levels of intracellular $\mathrm{Ca}^{2+}$ that could potentially interfere with the proper functioning of the rod photoreceptors, resulting in the CSNB phenotype (26). Concerning the frameshift disease-causing variant, it is predicted to change the last amino acid of the protein and to elongate the protein by 29 novel amino acids. It is likely that the elongated protein presents structural changes which may alter the cation binding affinity or the interaction with other proteins, influence its intracellular localization or modify its stability. It has been shown that SLC24A1interacts with the $\alpha$-subunit of cGMPgated channel (52). Whether the extended SLC24A1 protein alters the interaction with the cGMP-gated channel needs to be tested. Alternatively, the elongated SLC24A1 protein may be mislocalized, leading to complete loss of function as found for other mutants implicated in CSNB $(53,54)$. Finally, the extended SLC24A1 protein may be misfolded and, thus, become subject to degradation. 
Here, we showed that NGS techniques such as WES and targeted NGS are unbiased methods to identify gene defects leading to CSNB, helping clinicians to classify the subtype of the disorder where clinical diagnosis is unclear. Moreover, we identified four novel disease causing variants in SLC24A1 in three different families, expanding the disease causing variants spectrum for this gene. This report is only the second one which documents $S L C 24 A 1$ defects leading to CSNB, considering the fact that the previous one described only one disease causing variant in one family. Together with the knock-out mouse model, this paper reinforces the assertion that $S L C 24 A 1$, when mutated, is implicated in the Riggs-type of CSNB. 
1. Zeitz C. Molecular genetics and protein function involved in nocturnal vision. Expert Rev Ophtalmol 2007: 2: 467-485.

2. Zeitz C, Robson AG, Audo I. Congenital stationary night blindness: An analysis and update of genotype-phenotype correlations and pathogenic mechanisms. Prog Retin Eye Res 2015: 45C: 58-110.

3. Schubert G, Bornschein H. [Analysis of the human electroretinogram]. Ophthalmologica 1952: 123: 396-413.

4. Miyake $\mathrm{Y}$, Yagasaki $\mathrm{K}$, Horiguchi $\mathrm{M}$ et al. Congenital stationary night blindness with negative electroretinogram. A new classification. Arch Ophthalmol 1986: 104: 1013-1020.

5. Audo I, Robson AG, Holder GE et al. The negative ERG: clinical phenotypes and disease mechanisms of inner retinal dysfunction. Surv Ophthalmol 2008: 53: 16-40.

6. Bech-Hansen NT, Naylor MJ, Maybaum TA et al. Mutations in NYX, encoding the leucine-rich proteoglycan nyctalopin, cause X-linked complete congenital stationary night blindness. Nat Genet 2000: 26: 319-323.

7. Pusch $\mathrm{CM}$, Zeitz $\mathrm{C}$, Brandau $\mathrm{O}$ et al. The complete form of $\mathrm{X}$-linked congenital stationary night blindness is caused by mutations in a gene encoding a leucine-rich repeat protein. Nat Genet 2000: 26: 324-327.

8. Dryja TP, McGee TL, Berson EL et al. Night blindness and abnormal cone electroretinogram ON responses in patients with mutations in the GRM6 gene encoding mGluR6. Proc Natl Acad Sci U S A 2005: 102: 4884-4889.

9. Zeitz $\mathrm{C}$, van Genderen M, Neidhardt $\mathrm{J}$ et al. Mutations in GRM6 cause autosomal recessive congenital stationary night blindness with a distinctive scotopic $15-\mathrm{Hz}$ flicker electroretinogram. Invest Ophthalmol Vis Sci 2005: 46: 4328-4335.

10. Audo I, Kohl S, Leroy BP et al. TRPM1 is mutated in patients with autosomalrecessive complete congenital stationary night blindness. Am J Hum Genet 2009: 85: 720729.

11. van Genderen MM, Bijveld MM, Claassen YB et al. Mutations in TRPM1 are a common cause of complete congenital stationary night blindness. Am J Hum Genet 2009: 85: 730-736.

12. Li Z, Sergouniotis PI, Michaelides M et al. Recessive mutations of the gene TRPM1 abrogate $\mathrm{ON}$ bipolar cell function and cause complete congenital stationary night blindness in humans. Am J Hum Genet 2009: 85: 711-719.

13. Audo I, Bujakowska K, Orhan E et al. Whole-exome sequencing identifies mutations in GPR179 leading to autosomal-recessive complete congenital stationary night blindness. Am J Hum Genet 2012: 90: 321-330.

14. Peachey NS, Ray TA, Florijn R et al. GPR179 is required for depolarizing bipolar cell function and is mutated in autosomal-recessive complete congenital stationary night blindness. Am J Hum Genet 2012: 90: 331-339.

15. Zeitz C, Jacobson SG, Hamel CP et al. Whole-exome sequencing identifies LRIT3 mutations as a cause of autosomal-recessive complete congenital stationary night blindness. Am J Hum Genet 2013: 92: 67-75.

16. Strom TM, Nyakatura G, Apfelstedt-Sylla E et al. An L-type calcium-channel gene mutated in incomplete X-linked congenital stationary night blindness. Nat Genet 1998: 19: 260-263.

17. Bech-Hansen NT, Naylor MJ, Maybaum TA et al. Loss-of-function mutations in a calcium-channel alpha1-subunit gene in Xp11.23 cause incomplete X-linked congenital stationary night blindness. Nat Genet 1998: 19: 264-267.

18. Zeitz C, Kloeckener-Gruissem B, Forster $U$ et al. Mutations in CABP4, the gene encoding the Ca2+-binding protein 4, cause autosomal recessive night blindness. Am J Hum Genet 2006: 79: 657-667.

19. Wycisk KA, Zeitz C, Feil $S$ et al. Mutation in the auxiliary calcium-channel subunit CACNA2D4 causes autosomal recessive cone dystrophy. Am J Hum Genet 2006: 79: 973977.

20. Riggs LA. Electroretinography in cases of night blindness. Am J Ophthalmol 1954: 38 : 70-78. 
21. Ueno $S$, Kondo M, Niwa $Y$ et al. Luminance dependence of neural components that underlies the primate photopic electroretinogram. Invest Ophthalmol Vis Sci 2004: 45: 10331040.

22. Dryja TP, Berson EL, Rao VR et al. Heterozygous missense mutation in the rhodopsin gene as a cause of congenital stationary night blindness. Nat Genet 1993: 4: 280283.

23. Dryja TP, Hahn LB, Reboul T et al. Missense mutation in the gene encoding the alpha subunit of rod transducin in the Nougaret form of congenital stationary night blindness. Nat Genet 1996: 13: 358-360.

24. Gal A, Xu S, Piczenik Y et al. Gene for autosomal dominant congenital stationary night blindness maps to the same region as the gene for the beta-subunit of the rod photoreceptor cGMP phosphodiesterase (PDEB) in chromosome 4p16.3. Hum Mol Genet 1994: 3: 323-325.

25. Gal A, Orth U, Baehr W et al. Heterozygous missense mutation in the rod cGMP phosphodiesterase beta-subunit gene in autosomal dominant stationary night blindness. Nat Genet 1994: 7: 551.

26. Riazuddin SA, Shahzadi A, Zeitz $C$ et al. A mutation in SLC24A1 implicated in autosomal-recessive congenital stationary night blindness. Am J Hum Genet 2010: 87: 523531.

27. Rao VR, Cohen GB, Oprian DD. Rhodopsin mutation G90D and a molecular mechanism for congenital night blindness. Nature 1994: 367: 639-642.

28. al-Jandal N, Farrar GJ, Kiang AS et al. A novel mutation within the rhodopsin gene (Thr-94-lle) causing autosomal dominant congenital stationary night blindness. Hum Mutat 1999: 13: 75-81.

29. Zeitz C, Gross AK, Leifert D et al. Identification and functional characterization of a novel rhodopsin mutation associated with autosomal dominant CSNB. Invest Ophthalmol Vis Sci 2008: 49: 4105-4114.

30. Naeem MA, Chavali VR, Ali S et al. GNAT1 associated with autosomal recessive congenital stationary night blindness. Invest Ophthalmol Vis Sci 2012: 53: 1353-1361.

31. Szabo V, Kreienkamp HJ, Rosenberg T et al. p.GIn200Glu, a putative constitutively active mutant of rod alpha-transducin (GNAT1) in autosomal dominant congenital stationary night blindness. Hum Mutat 2007: 28: 741-742.

32. Manes G, Cheguru P, Majumder A et al. A truncated form of rod photoreceptor PDE6 beta-subunit causes autosomal dominant congenital stationary night blindness by interfering with the inhibitory activity of the gamma-subunit. PLoS One 2014: 9: e95768.

33. Wycisk KA, Budde B, Feil $S$ et al. Structural and functional abnormalities of retinal ribbon synapses due to Cacna2d4 mutation. Invest Ophthalmol Vis Sci 2006: 47: 3523-3530.

34. Audo I, Bujakowska KM, Leveillard T et al. Development and application of a nextgeneration-sequencing (NGS) approach to detect known and novel gene defects underlying retinal diseases. Orphanet J Rare Dis 2012: 7: 8.

35. Neveling K, Feenstra I, Gilissen $\mathrm{C}$ et al. A post-hoc comparison of the utility of sanger sequencing and exome sequencing for the diagnosis of heterogeneous diseases. Hum Mutat 2013: 34: 1721-1726.

36. El Shamieh S, Neuille M, Terray A et al. Whole-exome sequencing identifies KIZ as a ciliary gene associated with autosomal-recessive rod-cone dystrophy. Am J Hum Genet 2014: 94: 625-633.

37. Bujakowska KM, Zhang Q, Siemiatkowska AM et al. Mutations in IFT172 cause isolated retinal degeneration and Bardet-Biedl syndrome. Hum Mol Genet 2015: 24: 230-242. 38. Boulanger-Scemama E, El Shamieh $S$, Demontant $V$ et al. Next-generation sequencing applied to a large French cone and cone-rod dystrophy cohort: mutation spectrum and new genotype-phenotype correlation. Orphanet J Rare Dis 2015: 10: 85.

39. Marmor MF, Fulton AB, Holder GE et al. ISCEV Standard for full-field clinical electroretinography (2008 update). Doc Ophthalmol 2009: 118: 69-77.

40. Nakamura $\mathrm{M}$, Ito $\mathrm{S}$, Piao $\mathrm{CH}$ et al. Retinal and optic disc atrophy associated with a CACNA1F mutation in a Japanese family. Arch Ophthalmol 2003: 121: 1028-1033. 
41. Wutz K, Sauer C, Zrenner E et al. Thirty distinct CACNA1F mutations in 33 families with incomplete type of XLCSNB and Cacna1f expression profiling in mouse retina. Eur $\mathrm{J}$ Hum Genet 2002: 10: 449-456.

42. Vinberg F, Wang T, Molday RS et al. A new mouse model for stationary night blindness with mutant Slc24a1 explains the pathophysiology of the associated human disease. Hum Mol Genet 2015: 24: 5915-5929.

43. Boycott KM, Pearce WG, Bech-Hansen NT. Clinical variability among patients with incomplete $X$-linked congenital stationary night blindness and a founder mutation in CACNA1F. Can J Ophthalmol 2000: 35: 204-213.

44. Altimimi HF, Schnetkamp PP. Na+/Ca2+-K+ exchangers (NCKX): functional properties and physiological roles. Channels (Austin) 2007: 1: 62-69.

45. Schnetkamp PP. The SLC24 Na+/Ca2+-K+ exchanger family: vision and beyond. Pflugers Arch 2004: 447: 683-688.

46. Reid DM, Friedel U, Molday RS et al. Identification of the sodium-calcium exchanger as the major ricin-binding glycoprotein of bovine rod outer segments and its localization to the plasma membrane. Biochemistry 1990: 29: 1601-1607.

47. Carrigan M, Duignan E, Humphries $P$ et al. A novel homozygous truncating GNAT1 mutation implicated in retinal degeneration. Br J Ophthalmol 2015: 0: 1-6.

48. Calvert PD, Krasnoperova NV, Lyubarsky AL et al. Phototransduction in transgenic mice after targeted deletion of the rod transducin alpha -subunit. Proc Natl Acad Sci U S A 2000: 97: 13913-13918.

49. Schnetkamp PP. The SLC24 gene family of $\mathrm{Na}(+) / \mathrm{Ca}(2)(+)-\mathrm{K}(+)$ exchangers: from sight and smell to memory consolidation and skin pigmentation. Mol Aspects Med 2013: 34: 455-464.

50. Winkfein RJ, Szerencsei RT, Kinjo TG et al. Scanning mutagenesis of the alpha repeats and of the transmembrane acidic residues of the human retinal cone $\mathrm{Na} / \mathrm{Ca}-\mathrm{K}$ exchanger. Biochemistry 2003: 42: 543-552.

51. Altimimi HF, Fung $\mathrm{EH}$, Winkfein $\mathrm{RJ}$ et al. Residues contributing to the $\mathrm{Na}(+)$-binding pocket of the SLC24 Na(+)/Ca(2+)-K(+) Exchanger NCKX2. J Biol Chem 2010: 285: 1524515255.

52. Schwarzer A, Schauf H, Bauer PJ. Binding of the cGMP-gated channel to the $\mathrm{Na} / \mathrm{Ca}-$ K exchanger in rod photoreceptors. J Biol Chem 2000: 275: 13448-13454.

53. Orhan E, Prezeau L, El Shamieh S et al. Further insights into GPR179: expression, localization, and associated pathogenic mechanisms leading to complete congenital stationary night blindness. Invest Ophthalmol Vis Sci 2013: 54: 8041-8050.

54. Zeitz $\mathrm{C}$, Forster $\mathrm{U}$, Neidhardt $\mathrm{J}$ et al. Night blindness-associated mutations in the ligand-binding, cysteine-rich, and intracellular domains of the metabotropic glutamate receptor 6 abolish protein trafficking. Hum Mutat 2007: 28: 771-780. 
Fig.1: SLC24A1 novel disease causing variants in CSNB (A) Corresponding pedigrees of patients with CSNB with SLC24Al disease causing variants and co-segregation analysis. Indexes patients are individual II.1 of Family 1, II.4 of Family 2 and IV.2 of Family 3. Patients included for whole-exome-sequencing (WES) are marked with a red arrow. Patients included for targeted next-generation sequencing (NGS) are marked with a black arrow. Patients used for co-segregation analysis by Sanger sequencing are marked with a blue arrow. Square symbol $=$ male, round symbol $=$ woman, filled symbol $=$ affected, unfilled symbol $=$ unaffected, double line $=$ consanguinity, barred $=$ deceased. (B) Sequence electropherograms of CSNB patients with SLC24A1 disease causing variants, their relatives and controls. The underlined letters correspond to modifications of sequence induced by the different disease causing variants. For Family 1, affected patient II.2 displays the same profile as his affected brother II.1 and the unaffected father I.1 displays the same profile as the unaffected mother I.2; for Family 2, the non-affected sister II.3 displays the same profiles as the non-affected mother I.2; for Family 3, the non-affected brother IV.1 displays the same profile as the control.

\section{Fig.2: Conservation and positioning of SLC24A1 disease causing variants (A)} Evolutionary conservation of Ser990 in other orthologs shown in green. Amino acid substitution is highlighted in red. The position of the amino acid is shown in black numbers. (B) Topological model of SLC24A1. Transmembrane domains (TM) are identified by numbers 0 to 10 . Cleavage of the signal peptide, including TM 0 , is illustrated by a cross. Two novel disease causing variants reside in TM 4 and 7, one localizes in the intracellular loop between the two predicted ion exchanger domains and the last one was identified at the C-terminus of the protein. The disease causing variant previously described resides in TM3. 
Fig.3: Fundus photograph and SD-OCT scan of CSNB patients with SLC24A1 disease causing variants (A) and (C) Fundus photographs of patient II.4 of Family 2 and patient IV.2 of Family 3, respectively (C) and (D) Normal SD-OCT scans in both the patients.

Fig.4: Full-field electroretinogram of patient II.4 of Family 2 and patient IV.2 of Family 3, as an example (A) Dark-adapted 0.01 ERG (B) Dark-adapted 3.0 ERG (C) Dark-adapted 3.0 oscillatory potentials (D) Light-adapted 3.0 ERG (E) Light-adapted 3.0 flicker ERG Scotopic responses are severely reduced for both patients. Patient II.4 of Family 2 shows relatively conserved photopic responses whereas photopic ERG is altered in patient IV.2 of Family 3.

Table 1: Amplitudes and implicit times of electroretinogram responses of patient II.4 of Family 2 and patient IV.2 of Family 3, as an example Amplitudes (in $\mu \mathrm{V}$ ) and implicit times (in ms) of a-and b-waves in both patients compared to 8 non-affected individuals (Controls). Values for controls are given as mean \pm standard deviation (SD). Amp: Amplitudes; IT: Implicit times; NR: Non-recordable.

Table S1: Non-disease causing variants identified by WES after filtering dbSNP: Single Nucleotide Polymorphism database; MAF: Minor Allele Frequency; EVS: Exome Variant Server; Eur.Am.: European American; Afr.Am.: African American; ExAC: Exome Aggregation Consortium; AFR: African; AMR: American; EAS: East Asian; SAS: South Asian; NFE: Non-Finnish European; FIN: Finnish; OTH: Other; SIFT: Sorting Intolerant from Tolerant; UCSC: UCSC Genome Browser.

Table S2: Novel disease causing variants identified in SCL24A1 (NM_004727.2) [MIM 603617] by WES and targeted NGS after filtering Unaff.: Unaffected; Aff.: Affected; Homo: Homozygous; Het: Heterozygous; NMD: Non-Mediated Decay; n.d.: not determined.

Table S3: Quality control of the WES 
Table S4: Quality control of the targeted NGS 
A

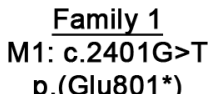

p.(Glu801*)

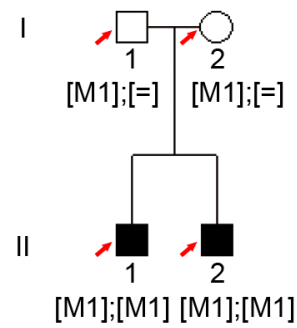

B

Family 1

II.1

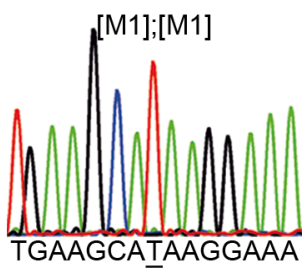

1.2

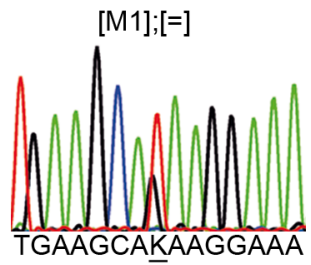

Control

[=];[=]

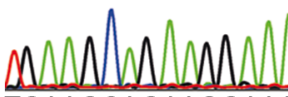

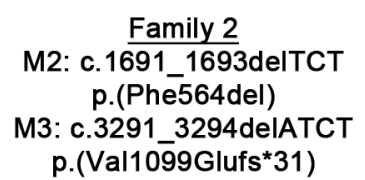
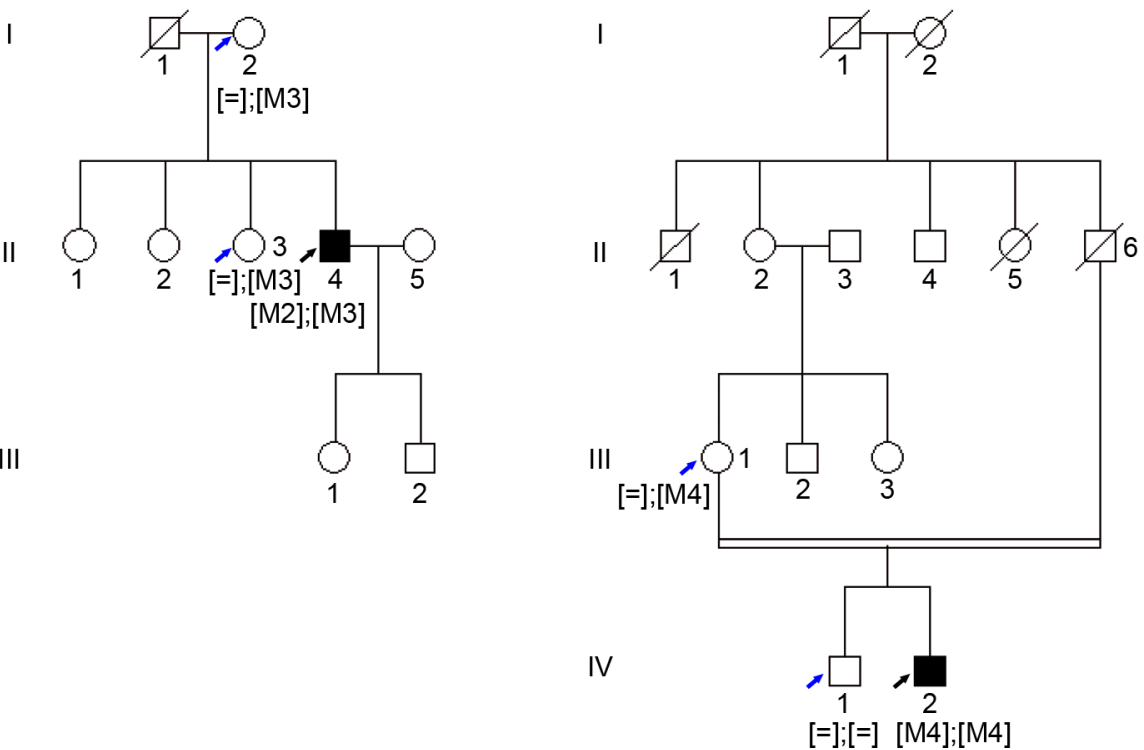

Family 2

II. 4

[M2];[=]

[=];[M3]

[M4];[M4]

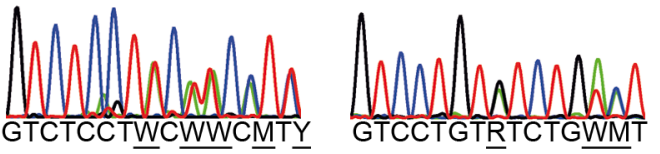

12

$[=] ;[=]$
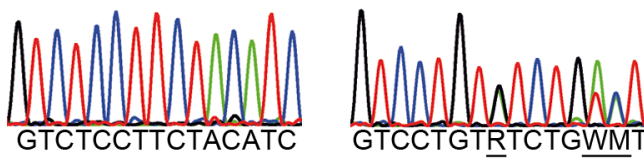

Control

$[=] ;[=]$

$[=] ;[=]$

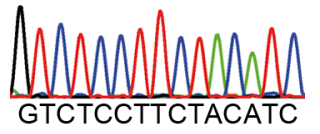

p.(Ser990Arg)

[=];[=] [M4];[M4]

$\underline{\text { Family } 3}$

IV. 2

\section{$\frac{\text { MMnamman }}{\text { CATCACCEGTGTGAT }}$}

III.1

[M4];[=]

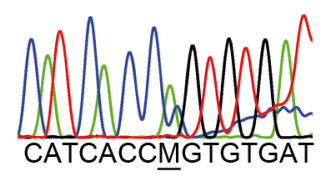

Control

[=];[=]

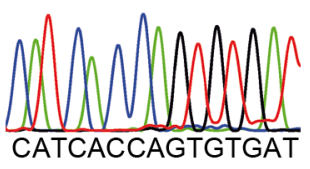


A

990

$R$

Human PDLITSVIVAR

Rhesus PDLITSVIVAR

Mouse PDLITSVIVAR

Cat PDLITSVIVAR

Elephant PDLITSVIVAR

Opossum PDLITSVIVAR

Chicken PDLITSVIVAR

Lizard PDLITSVIVAR

X_tropicalis PDLITSVIVAR

Stickleback PDLITSVIVAR

Zebrafish PDLITSVIVAR

B

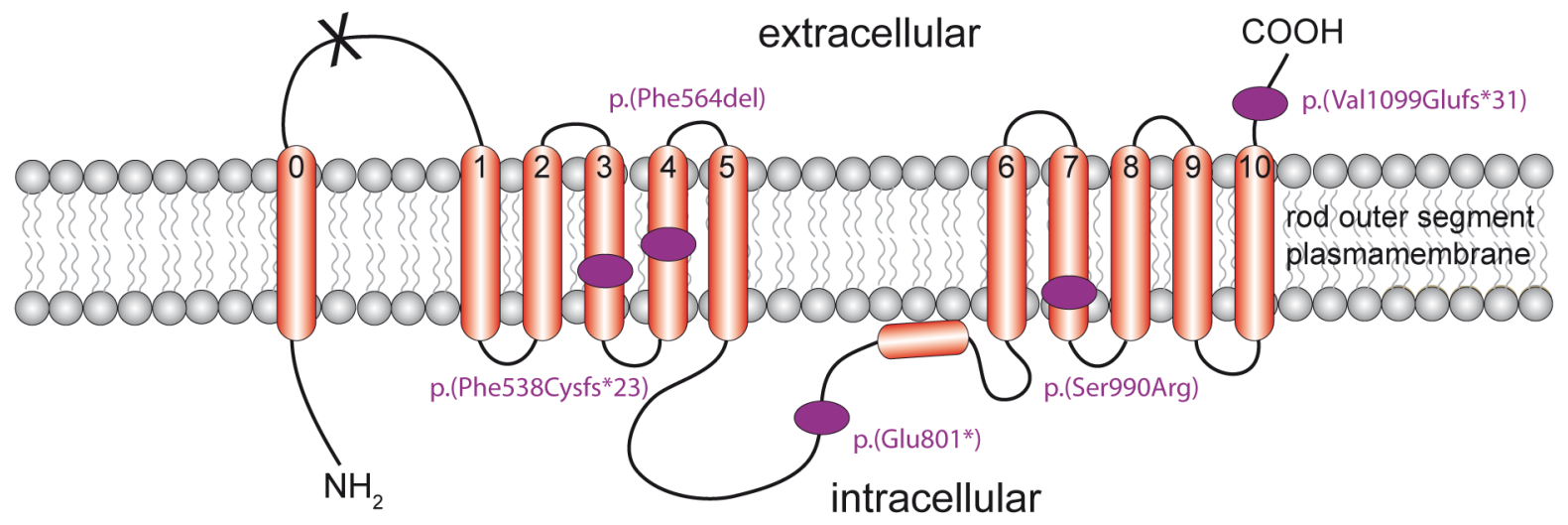


II. 4 of Family 2
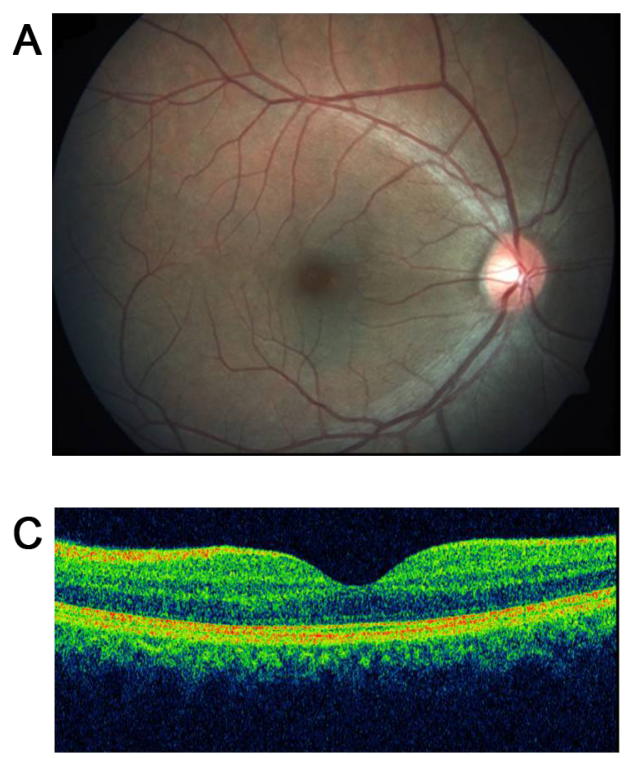

IV.2 of Family 3

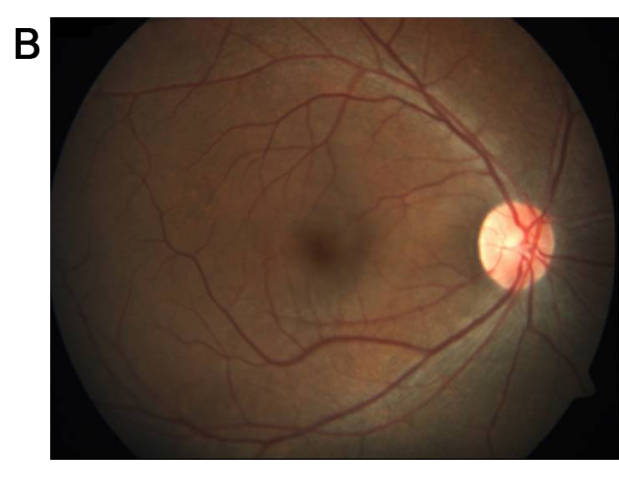

D

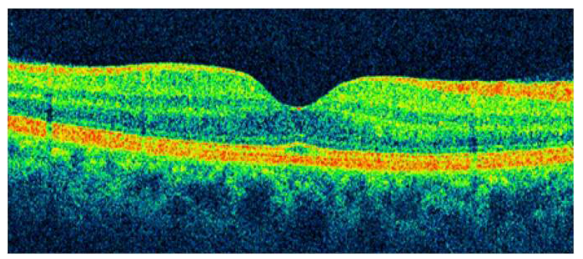


Control

A

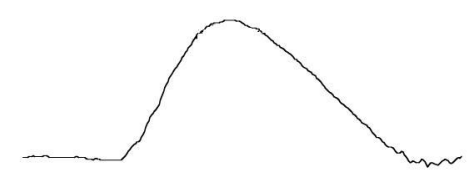

B

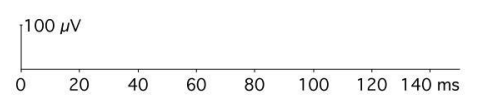

C
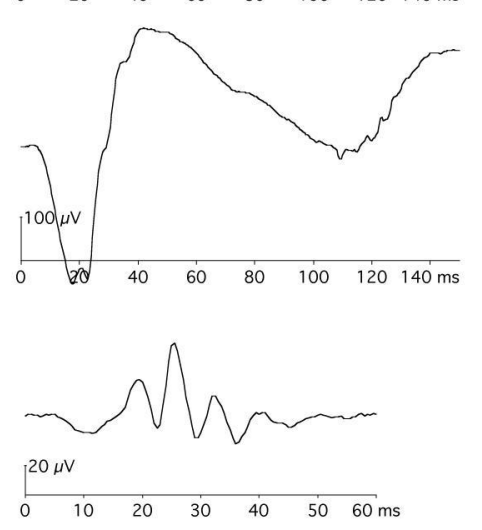

D
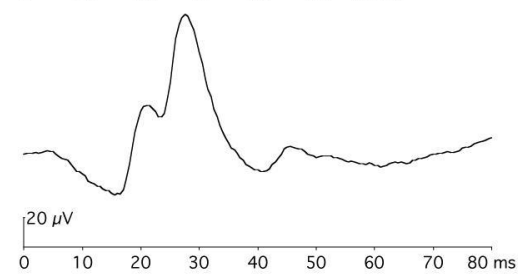

E

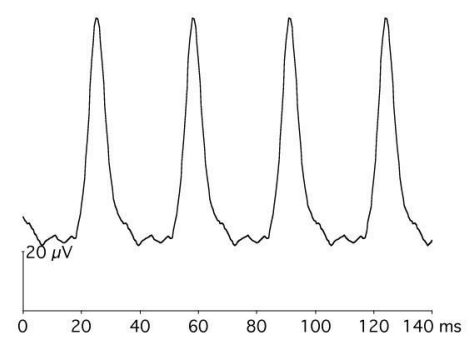

II. 4 of Family 2
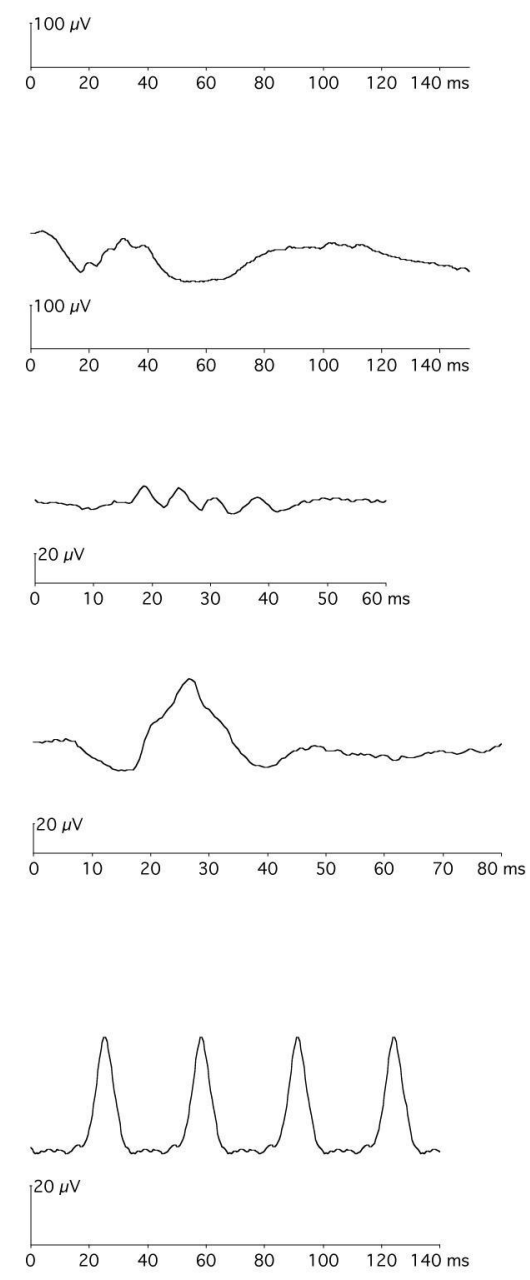

IV.2 of Family 3
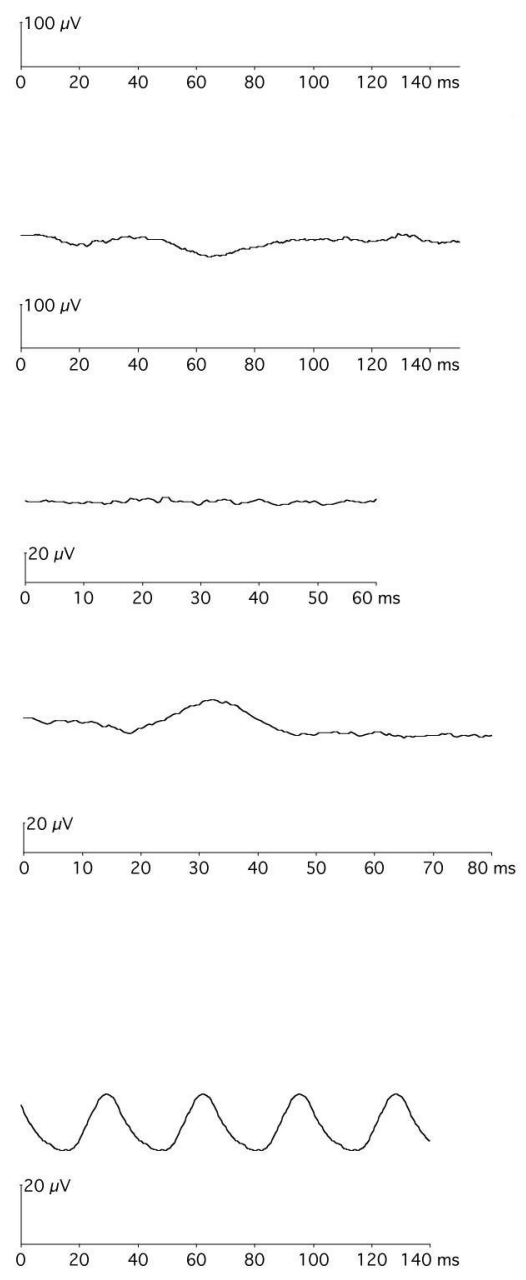


\begin{tabular}{|c|c|c|c|c|c|c|c|c|c|c|c|c|}
\hline & \multicolumn{2}{|c|}{$\begin{array}{l}\text { Dark-adapted } \\
\text { 0.01 ERG } \\
\text { b-wave }\end{array}$} & \multicolumn{2}{|c|}{$\begin{array}{l}\text { Dark-adapted } \\
\text { 3.0 ERG } \\
\text { a-wave }\end{array}$} & \multicolumn{2}{|c|}{$\begin{array}{l}\text { Dark-adapted } \\
\text { 3.0 ERG } \\
\text { b-wave }\end{array}$} & \multicolumn{2}{|c|}{$\begin{array}{l}\text { Light-adapted } \\
\text { 3.0 ERG } \\
\text { a-wave }\end{array}$} & \multicolumn{2}{|c|}{$\begin{array}{l}\text { Light-adapted } \\
\text { 3.0 ERG } \\
\text { b-wave }\end{array}$} & \multicolumn{2}{|c|}{$\begin{array}{l}\text { Light-adapted } \\
\text { 30Hz flicker ERG } \\
\text { b-wave }\end{array}$} \\
\hline & $\operatorname{Amp}(\boldsymbol{\mu V})$ & IT (ms) & $\operatorname{Amp}(\mu \mathrm{V})$ & IT (ms) & $\operatorname{Amp}(\mu \mathrm{V})$ & IT (ms) & $\operatorname{Amp}(\boldsymbol{\mu V})$ & IT (ms) & $\operatorname{Amp}(\mu \mathrm{V})$ & IT (ms) & $\operatorname{Amp}(\mu \mathrm{V})$ & IT (ms) \\
\hline Controls $(\mathrm{n}=8)$ & $\begin{array}{l}297.9 \\
\pm 83.4\end{array}$ & $\begin{array}{l}63.13 \\
\pm 3.29 \\
\end{array}$ & $\begin{array}{l}226.4 \\
\pm 54.6\end{array}$ & $\begin{array}{l}16.88 \\
\pm 0.52\end{array}$ & $\begin{array}{l}479.4 \\
\pm 116.1\end{array}$ & $\begin{array}{l}44.94 \\
\pm 2.35 \\
\end{array}$ & $\begin{array}{l}25.3 \\
\pm 5.0\end{array}$ & $\begin{array}{l}16.25 \\
\pm 0.93 \\
\end{array}$ & $\begin{array}{l}105.6 \\
\pm 26.7\end{array}$ & $\begin{array}{l}27.31 \\
\pm 0.88\end{array}$ & $\begin{array}{l}65.5 \\
\pm 10.6 \\
\end{array}$ & $\begin{array}{l}25.19 \\
\pm 0.80\end{array}$ \\
\hline II.4 of Family 2 & 6.6 & 50.0 & 88.9 & 17.0 & 57.6 & 35.0 & 19.3 & 16.5 & 62.7 & 26.5 & 39.8 & 25.5 \\
\hline IV.2 of Family 3 & NR & NR & 26.1 & 22.5 & 17.3 & 43.0 & 11.0 & 17.5 & 23.4 & 31.5 & 19.3 & 29.0 \\
\hline
\end{tabular}

Table 1 